\title{
«ll ne faut pas avoir peur de se tromper»
}

Le Dr Thomas Heuberger a été président de l'Assemblée des délégués de 2007 à 2013. Grâce à une bonne connaissance des dossiers, il a su diriger les séances avec compétence et écouter les différents avis tout en restant ouvert aux nouvelles idées. Il a quitté ses fonctions à la fin de l'année dernière, une occasion pour lui de revenir sur son activité, son vécu et ce qui lui tenait à cœur.

Interview:

Jacqueline Wettstein

Cheffe de la Communication

Correspondance:

Jacqueline Wettstein FMH

Division Communication

Elfenstrasse 18

CH-3000 Berne 15

Tél. 0313591150

Fax 0313591112

kommunikation[at]fmh.ch
L'Assemblée des délégués de la FMH (AD) fait le lien entre la Chambre médicale et le Comité central. Vous avez étéle premier à la présider. Qu'est-ce qui vous a particulièrement intéressé et que souhaitiez-vous y réaliser?

Mon ambition était de participer à une nouvelle structure de la FMH dans le but de contribuer à un meilleur ancrage de la politique professionnelle au sein de la Chambre médicale et du corps médical dans son ensemble, ainsi que d'offrir au Comité central une base de réflexion plus large pour ses décisions politiques.

L'Assemblée des délégués existe depuis 6 ans maintenant. Comment a-t-elle évolué et où se positionnera-t-elle dans 10 ans?

Les hésitations et la retenue du début ont fait place à une plus grande confiance. Nos arguments sont devenus plus percutants. Il me semble que c'est aussi l'avis du Comité central qui estime l'Assemblée des délégués pour son aide aux prises de décisions. Où se positionnera-t-elle dans 10 ans? Peut-on se projeter si loin en avant dans le domaine de la santé? L'AD sera certainement totalement intégrée dans les démarches et les réflexions des médecins en politique de la santé. Elle développera ses propres idées et projets, et sera le sparring-partner incontournable du Comité central.

Quelle est sa plus grande force? Et quelles améliorations faudrait-il entreprendre?

Sa plus grande force est certainement qu'elle est consciente de jouer un rôle important en politique de la santé en tant que représentante des médecins. Elle donne également plus de poids aux activités politiques de la FMH. De plus, les discussions politiques au sein de l'AD ne poursuivent pas uniquement des intérêts particuliers mais touchent toutes les disciplines et ce dans l'intérêt de l'ensemble du corps médical.

Mais malgré tout, des améliorations doivent être faites notamment en ce qui concerne le flux d'informations réciproques entre l'AD et le Comité

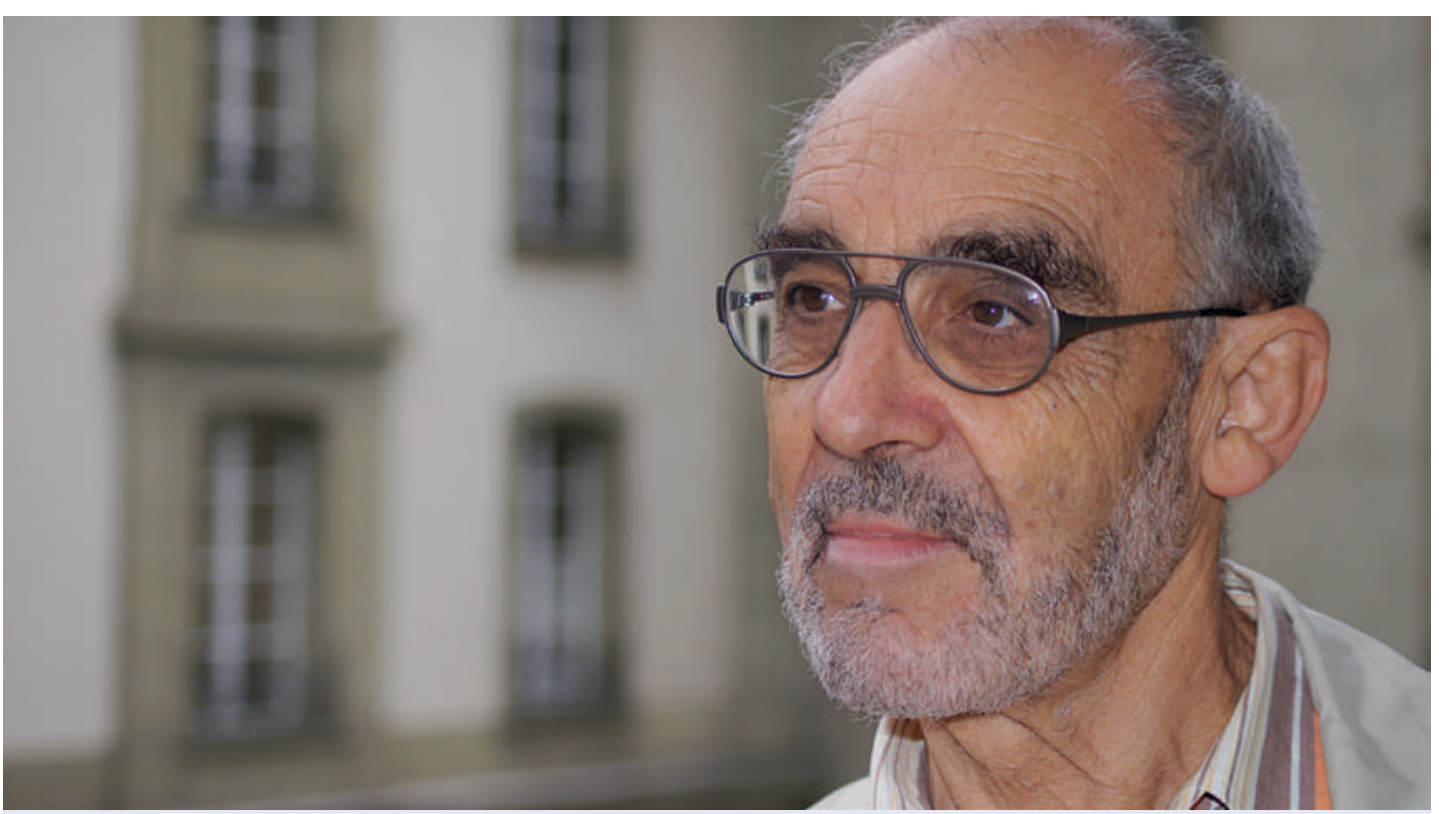

Thomas Heuberger, premier président de l'Assemblée des délégués, passe le témoin et laisse une assemblée en confiance. 


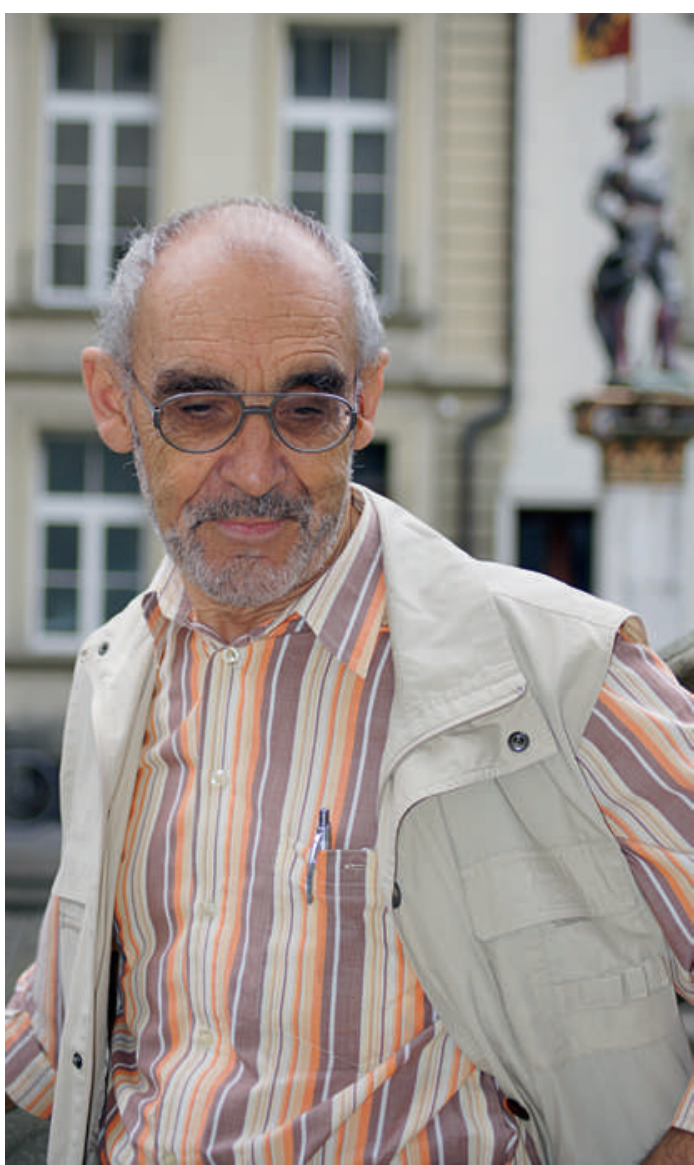

Une des tâches les plus passionnantes consiste à diriger des débats animés et parfois controversés.

central d'une part, et l'AD et la base d'autre part, en particulier sur les sujets de politique de la santé. Il reste encore des efforts à fournir pour garantir un meilleur ancrage des délégués dans la politique de leurs organisations cantonales et des organisations faîtières.

Quelles ont été vos premières impressions lors de votre accès à la présidence et quel regard portiez-vous sur l'AD fin 2013?

A mes débuts, j'étais prudent et j'avais beaucoup de respect devant la tâche à accomplir. Et puis l'enthousiasme et le plaisir à débattre et à traiter les différents sujets ont pris le dessus. Après les séances, j'étais quand même souvent sous tension en raison des travaux de préparation et d'organisation. Mais je me suis aussi toujours senti amicalement soutenu par les délégués et les membres du Comité central.

Comment s'est déroulée la collaboration avec le Comité central?

Elle a été excellente, ouverte et informative. Il m'a semblé très important de participer aux séances du Comité central, de pouvoir discuter de l'ordre du jour et prendre connaissance des affaires à traiter ensuite éventuellement à l'Assemblée des délégués.
Entre 2007 et 2013, Thomas Heuberger a été le premier président de l'Assemblée des délégués de la FMH. Après avoir obtenu son titre de spécialiste en médecine interne générale en 1980, il a exercé comme médecin de famille dans son propre cabinet. En parallèle, il a obtenu les attestations de formation complémentaire en acupuncture et médecine traditionnelle chinoise ainsi qu'en pratique du laboratoire au cabinet médical. Il s'est engagé très tôt en politique professionnelle: en 1984, il a été élu au Comité de la Société des médecins du canton de Berne et depuis 2000, il est membre de la Chambre médicale. D'autres fonctions sont venues s'ajouter à celle de président de l'Assemblée des délégués de la FMH: depuis 2011, il préside en effet l'ORTRA Formation professionnelle des assistantes médicales en tant que délégué de la FMH, et depuis 2012, la Commission suisse pour le développement professionnel et la qualité des assistantes médicales. Hormis son engagement auprès de l'Ecole professionnelle bernoise pour la profession d'assistante médicale (be-med AG) et de l'Ecole professionnelle de l'Oberland bernois NOSS à Spiez, il participe à la politique bernoise en tant que représentant des Verts: c'est ainsi qu'il a été pendant neuf ans membre du Conseil communal d'Oberhofen, qu'il a représenté les Verts pendant treize ans au Grand Conseil du canton de Berne et qu'il a été pendant deux ans vice-président des Verts bernois.

Le rôle de président de l'AD est complexe. Sur quelles expériences avez-vous pu vous appuyer?

L'expérience politique acquise dans les assemblées communales et au sein du parlement cantonal m'a beaucoup aidé pour préparer et diriger les débats. Mon intérêt à travailler politiquement au service de la collectivité, et dans le cas de l'AD au service des médecins, a été ma principale motivation, hormis mon plaisir à débattre.

Quel est votre plus beau souvenir et pourquoi?

La partie la plus difficile, mais aussi la plus passionnante, a été de devoir souvent faire preuve de retenue dans mes opinions personnelles, parfois percutantes, afin de garantir la neutralité des débats.

J'ai toujours trouvé passionnant de discuter d'un sujet particulier avec des spécialistes externes ou des représentants politiques qui n'étaient pas du même avis que moi.

Et si c'était à refaire, que feriez-vous différemment? L'Assemblée des délégués et les débats qui s'y sont tenus vont me manquer. Ma manière de préparer les sujets aurait souvent mérité d'être plus précise et compréhensible. Autrement dit, j'aurais dû y consacrer encore plus de temps au détriment de mon acti- 


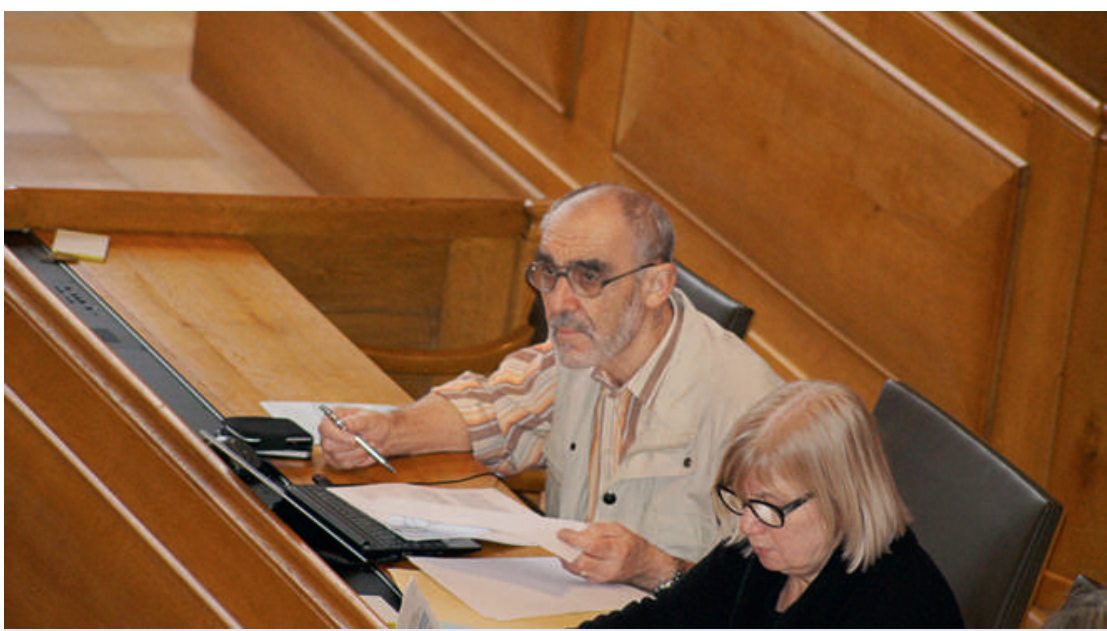

Session au Grand Conseil bernois: «Grâce à la politique, je peux rendre à la communauté ce qu'elle m'apporte.» les mandats de l'actuel président de la FMH. J'ai également été assesseur et j'ai fait le lien avec la politique cantonale grâce à mon siège au Grand Conseil bernois.

La politique me permet de rendre à la société un peu de ce qu'elle m'a donné. C'est le cas aussi bien pour la politique en général que pour la politique professionnelle. Le travail politique est particulièrement intéressant une fois qu'on s'y est familiarisé et que l'on peut développer ses propres projets. Participer au débat politique permet d'évoluer dans un contexte social différent de celui du cabinet, de nouer de nouveaux contacts et d'élargir son horizon, ce qui est un changement bienvenu dans le quotidien.

Comme vous avez désormais atteint la durée maximale possible dans cette fonction, vous avez passé le témoin à Peter Wiedersheim qui dirige l'Assemblée des délégués depuis 2014. Quelles sont les qualités dont il devra faire preuve?

Avoir un bon réseau, de la créativité politique, de la persévérance et un esprit conceptuel. Des qualités dont Peter Wiedersheim dispose déjà, c'est donc un successeur parfait! Selon moi, il est également important de faire preuve de courage face à des propositions et à des idées non conventionnelles: il ne faut pas avoir peur de se tromper.

Qu'est-ce qui vous fascine tant en politique?

En 1984, Walter Flury, alors président de la Société des médecins du canton de Berne, m'a invité à participer aux travaux du comité et j'ai attrapé le virus. Depuis lors, je n'ai pratiquement jamais cessé d'être actif dans différentes fonctions au sein du comité, dont onze ans en qualité de vice-président pendant

Fondée en 2007, l'Assemblée des délégués de la FMH assure le lien entre le Comité central et la Chambre médicale. Elle a été conçue pour remplacer l'ancienne Conférence des présidents et ses quelque 100 membres dans le but de disposer d'un organe plus petit et donc mieux à même de participer activement à la politique de santé. Avec ses 33 représentants des organisations faîtières confirmés par la Chambre médicale, l'Assemblée des délégués examine de manière autonome toutes les questions importantes en matière de politique de santé et professionnelle et les adopte à l'intention de la Chambre médicale. Par ailleurs, elle définit la ligne politique suivie par la FMH lors de votations populaires, du lancement d'une initiative ou d'un référendum.
Que souhaiteriez-vous transmettre à votre successeur? Et aux membres de l'Assemblée des délégués?

De la persévérance, de la créativité et du temps! Et savoir rester fidèle à ses convictions sans pour autant se fermer aux nouvelles idées. En tant que président, il faut parfois savoir se mettre en porte-à-faux.

Partenaires importants en politique de santé, les délégués doivent faire preuve de confiance et d'estime de soi. En outre, ils doivent entretenir le lien avec les membres qu'ils représentent: leur rôle d'intermédiaire consiste à faire suivre les informations mais aussi à créer le lien entre les processus politiques et l'opinion de leur base.

Une période de travail intense touche désormais à sa fin. Quels sont vos plans pour la suite?

Je compte poursuivre mon travail au cabinet pendant un moment, en réduisant un peu mon taux d'occupation. Par ailleurs, je continuerai à œuvrer en faveur de la formation de base et continue des assistantes médicales pour le compte de la FMH. Enfin, les passe-temps typiques d'un retraité (à temps partiel) m'attendent: famille, amis, temps libre, sport, lecture, hobbies - je n'aurai donc pas souvent l'occasion de m'ennuyer. 